\title{
The emerging role of microRNAs and long noncoding RNAs in drug resistance of hepatocellular carcinoma
}

\author{
Ling Wei ${ }^{1}$, Xingwu Wang ${ }^{1}$, Liyan Lv', Jibing Liu ${ }^{1,3}$, Huaixin Xing ${ }^{1}$, Yemei Song ${ }^{1}$, Mengyu Xie ${ }^{1}$, Tianshui Lei ${ }^{1}$, \\ Nasha Zhang ${ }^{2^{*}}$ and Ming Yang ${ }^{1 *}$ (D)
}

\begin{abstract}
Hepatocellular carcinoma (HCC) is the fifth most common malignancy worldwide and the second most lethal human cancer. A portion of patients with advanced HCC can significantly benefit from treatments with sorafenib, adriamycin, 5-fluorouracil and platinum drugs. However, most HCC patients eventually develop drug resistance, resulting in a poor prognosis. The mechanisms involved in HCC drug resistance are complex and inconclusive. Human transcripts without protein-coding potential are known as noncoding RNAs (ncRNAs), including microRNAs (miRNAs), small nucleolar RNAs (snoRNAs), long noncoding RNAs (IncRNAs) and circular RNA (circRNA). Accumulated evidences demonstrate that several deregulated miRNAs and IncRNAs are important regulators in the development of HCC drug resistance which elucidates their potential clinical implications. In this review, we summarized the detailed mechanisms by which miRNAs and IncRNAs affect HCC drug resistance. Multiple tumorspecific miRNAs and IncRNAs may serve as novel therapeutic targets and prognostic biomarkers for HCC.
\end{abstract}

Keywords: Hepatocellular carcinoma, Drug resistance, Long non-coding RNA, microRNA

\section{Background}

Hepatocellular carcinoma (HCC) is the fifth most prevalent malignancy worldwide, accounting for approximately $90 \%$ of primary liver cancer, characterized by high mortality, recurrence, metastasis and poor prognosis [1]. Due to the lack of symptoms in the early disease stages, most patients were diagnosed at the advanced disease stages with metastasis and poor hepatic reserve, thus losing the opportunity of curative resection. In the last decade, improved drug therapy agents have significantly prolonged the survival of HCC patients with advanced diseases. The commonly used therapeutic regimens include sorafenib, adriamycin (ADM), 5-fluorouracil (5-FU), platinum-containing anti-cancer drugs, camptothecin and gemcitabine [2-4]. However, the

\footnotetext{
*Correspondence: wownseva@126.com; aaryoung@yeah.net

2Department of Radiation Oncology, Shandong Cancer Hospital and Institute,

Shandong First Medical University and Shandong Academy of Medical

Sciences, Jinan 250117, Shandong Province, China

'Shandong Provincial Key Laboratory of Radiation Oncology, Cancer

Research Center, Shandong Cancer Hospital and Institute, Shandong First

Medical University and Shandong Academy of Medical Sciences, Jinan

250117, Shandong Province, China

Full list of author information is available at the end of the article
}

acquisition of multi-drug resistance (MDR) to these agents is the Achilles' Heel in clinical oncology, and may result in a poor prognosis. Intrinsic or acquired drug resistance is defined as the resistance of malignant cells to different structurally and functionally unrelated anticancer drugs. The mechanisms involved in HCC drug resistance are complex and include the increased expression of drug efflux transporters that recognize and pump out anticancer drugs out of tumor cells, redistribution of intracellular accumulation of agents, inactivation of apoptosis signaling pathways, enhanced DNA damage repair capacity, accelerated drug metabolism and activation of cancer stem cells (CSCs) [5-7]. Up to now, however, the precise mechanisms underlying HCC drug resistance remain to be investigated.

Human transcripts without protein-coding potential are known as noncoding RNAs (ncRNAs). According to the length and shape, ncRNAs can be subdivided into the following types: tiny/short ncRNAs, long ncRNAs (lncRNAs) larger than 200 nucleotides (nt) and circular RNA (circRNAs). There are various small ncRNAs, such as microRNAs (miRNAs), PIWI-interacting RNAs (piRNAs), small nucleolar RNAs (snoRNAs) and small nuclear RNAs 
(snRNAs) [8-16]. NcRNAs were once considered as the "dark matter" or "by-products" during gene transcription. It has been found that ncRNAs are involved in various cellular functions, including proliferation, apoptosis and the cell cycle progression $[17,18]$. For instance, snoRNAs are essential for guidance of chemical modifications of other RNAs, whereas tRNAs and rRNAs are critical for protein translation. MiRNAs and lncRNAs play a vital role in regulating gene expression via their fine regulation at various levels, including transcription, translation and protein functions. Multiple miRNAs and lncRNAs not only can serve as biomarkers for diagnosis of multiple cancers, but also are involved in drug resistance [19-26]. Lots of ncRNAs have been found to be abnormally expressed in HCC and to be associated with the invasion, metastasis, drug resistance and radioresistance of HCC cells [19-26]. Among the abnormally expressed ncRNAs, multiple miRNAs and lncRNAs have been found to play essential roles in HCC drug resistance.

In this review, we systematically summarize the literatures on miRNAs and lncRNAs modulating HCC drug resistance as well as the underlying mechanisms, thereby providing insight into the role of ncRNAs as putative biomarkers and/or therapeutic targets of $\mathrm{HCC}$ in the future.

\section{MiRNAs and therapy resistance}

Currently, drug therapy for HCC includes targeted therapy, chemotherapy and immunotherapy. Molecular targeted drugs include sorafenib, regorafenib, lenvatinib and tivantinib. Chemotherapeutic drugs commonly used in clinics are adriamycin, 5-FU, cisplatin, and oxaliplatin. As for immunotherapy for HCC, it is a novel management choice and principally includes immune checkpoint blockers/monoclonal antibodies against the programmed cell death protein 1 (PD-1), PD-1 ligand (PD-L1) and receptor cytotoxic T lymphocyte antigen-4 (CTLA-4), such as nivolumab, pembrolizumab, MED14736, ipilimumab and tremelimumab.

As a class of endogenous, small, single-stranded ncRNAs of 19-24 nucleotides (nt) in length, miRNAs could bind to the 3 '-untranslated regions ( 3 '-UTRs) of target messenger RNAs (mRNAs) and suppress their expression and/or prohibit the translation. miRNAs are commonly involved in cellular differentiation, proliferation, death, angiogenesis and metabolic stress responses [27-29]. Dysregulated miRNAs act as oncogenes or tumor suppressors in the development and progression of many cancers, including HCC [30-32]. Interestingly, significantly altered miRNA expression have been found in a variety of drug-resistant HCC cells compared to those in drug-sensitive cells, suggesting that prediction of efficacy of different drugs using various miRNAs may promote individualized HCC therapy [25,33-36]. miRNAs can regulate immune checkpoint molecules expression in tumor microenvironment [37]. However, it is still unclear how miRNAs are involved in resistance to immune checkpoint blockers currently. Moreover, emerging evidences indicate that multiple miRNAs involved in sorafenib resistance. By contrast, few miRNAs have been found to be participated in resistance to other novel targeted drugs.

\section{MiRNAs and resistance to sorafenib}

Sorafenib, an oral multikinase inhibitor, was initially demonstrated to suppress proliferation and angiogenesis by targeting BRAF, Raf- 1 , Flt3, VEGFR-2/3 and PDGFR- $\beta$. Subsequently, it was also indicated that sorafenib could target signaling pathways independent of Raf, especially pathways regulating apoptosis and cell cycle progression [38-40]. As an FDA-approved standard targeted therapy agent for HCC, sorafenib showed survival benefits in advanced $\mathrm{HCC}$ patients worldwide. However, most patients eventually progressed to drug resistance diseases. Currently, the mechanisms involved in sorafenib resistance include activation of EGFR, c-Jun and/or AKT, microenvironmental hypoxia, epithelial-mesenchymal transition (EMT), activation of CSCs, resistance to apoptosis, cell cycle dysregulation, autophagy, and the abnormal expression of miRNAs and lncRNAs [36, 41-48]. A variety of miRNAs have been

Table 1 MiRNAs and sorafenib resistance in HCC

\begin{tabular}{|c|c|c|c|}
\hline MiRNAs & Expression $^{1}$ & Pathway & Reference \\
\hline miR-21 & up-regulated & PTEN/Akt & [49] \\
\hline miR-93 & up-regulated & PTEN; CDKN1A & [50] \\
\hline miR-216a /217 & up-regulated & PTEN; SMAD7 & {$[51]$} \\
\hline miR-181a & up-regulated & RASSF1 & {$[52]$} \\
\hline \multirow[t]{2}{*}{ miR-494 } & up-regulated & PTEN & {$[53]$} \\
\hline & & mTOR & {$[45]$} \\
\hline miR-221 & up-regulated & caspase-3 & {$[46]$} \\
\hline \multirow[t]{5}{*}{ miR-122 } & down-regulated & IGF-1R & {$[54]$} \\
\hline & & IGF-1R; SRF; ADAM10 & {$[55]$} \\
\hline & & PDK4 & {$[56]$} \\
\hline & & SLC7a1 & {$[57]$} \\
\hline & & GALNT10 & {$[58]$} \\
\hline miR-34a & down-regulated & $\mathrm{BCl}-2$ & {$[59]$} \\
\hline miR-27b & down-regulated & P53 & {$[60]$} \\
\hline let-7 & down-regulated & $B C L-X L$ & {$[61]$} \\
\hline miR-193b & down-regulated & MCL1 & {$[62,63]$} \\
\hline miR-486 & down-regulated & CLDN10; CITRON & {$[64]$} \\
\hline miR-367-3p & down-regulated & Androgen receptor & {$[65]$} \\
\hline miR-338-3p & down-regulated & HIF-1a & {$[43]$} \\
\hline miR-137 & down-regulated & ANT2 & {$[66]$} \\
\hline miR-142-3p & down-regulated & ATG5/ATG16L1 & {$[36]$} \\
\hline miR-7 & down-regulated & TYRO3 & {$[67]$} \\
\hline
\end{tabular}

${ }^{1}$ miRNAs either up-regulated or down-regulated in sorafenib resistant hepatocellular carcinoma cells

Note: This table shows 18 miRNAs, their expression level and potential targets in sorafenib resistance of hepatocellular carcinoma 
reported to be involved in sorafenib resistant in HCC (Table 1).

Several oncogenic miRNAs can promote sorafenib resistance [45, 49-53]. For example, miR-93, miR-216a and miR217 could confer sorafenib resistance by targeting cell cycle protein-dependent kinase $1 A(C D K N 1 A)$ and modulating apoptosis as well as the TGF- $\beta$ signaling $[50,51]$. Additionally, miR-181 can trigger sorafenib resistance by targeting and suppressing Ras association domain family member 1 (RASSF1) [52]. Exogenous expression of miR-494 has been found to increase resistance of HCC to sorafenib via targeting PTEN and activating the mTOR signaling. In animal models, sorafenib combined with anti-miR-494 potentiated the sensitivity of HCC to sorafenib, suggesting that miR-494 is a possible therapeutic target of advanced HCC $[45,53]$. It was also found that miR-221 potentiates sorafenib resistance by suppressing caspase- 3 mediated apoptosis in vivo. Moreover, its expression in serum has been found to be significantly lower in sorafenib responders compared to nonresponders, suggesting that miR-221 may be a candidate biomarker to predict responders to sorafenib [46].

Meanwhile, multiple tumor suppressor miRNAs can reverse sorafenib resistance in HCC. MiR-122, for instance, has been found to be the most highly expressed miRNA in normal liver and remarkably reduced in sorafenibresistant HCC cells. MiR-122 could promote sorafenib sensitivity of HCC cells through targeting and suppressing insulin-like growth factor 1 receptor (IGF-1R), serum response factor (SRF), depolymerization and metalloproteinase domain-containing protein 10 (ADAM10), pyruvate dehydrogenase kinase 4 (PDK4), solute carrier family 7 member 1 (SLC7A1) and peptide N-acetyl N-acetylgalactosaminyl transferase 10 (GALNT10) [54-58]. Similarly, miR-34a has been found to reverse the tolerance of HCC cells to sorafenib via silencing $B C L-2$ [59]. MiR-27b, let-7 and miR-193b which are curial regulators of apoptosis, also have been demonstrated to enhance sensitivity of HCC cells to sorafenib by silencing P53, Bcl-XL and/or myeloid leukemia cell differentiation protein (MCL1), respectively [60-63]. In addition, the tumor suppressor miR-486 and miR-367-3p have been shown to promote sorafenib sensitivity by inhibiting CITRON, claudin 10 (CLDN10) and androgen receptor $(A R)[64,65]$. It has been reported that miR-338-3p can sensitize HCC cells to sorafenib by silencing hypoxia inducing factor $1 \alpha(H I F-1 \alpha)$ in vitro and in vivo [43]. MiR-137 has been found to be significantly down-regulated in sorafenib-resistant Huh7-R HCC cells. Exogenous miR-137 can promote sorafenib sensitivity and inhibit cancer initiation cell $(\mathrm{CIC})$ phenotype through targeting adenine nucleotide transporter 2 (ANT2) [66]. Ectopic expression of miR-142-3p, a novel autophagy regulator, can sensitize HCC cells to sorafenib by silencing autophagy related 5 (ATG5) and autophagy related 16-like 1 (ATG16L1) and, thus, promote autophagy induced by sorafenib [36]. In addition, the tumor suppressor miR-7 has been found to promote sorafenib sensitivity in HCC cells through silencing expression of TYRO3, a member of TYRO3-AXL-MER family of receptor tyrosine kinases [67].

\section{MiRNAs and resistance to adriamycin}

Adriamycin, an anthracycline antibiotic and non-specific periodic drug, is a strong inhibitor of DNA and RNA synthesis in tumor cells during proliferation. Adriamycin could diffuse into the nucleus of HCC cells, interact with DNA and eventually induce apoptosis. There are some known mechanisms for development of adriamycin resistance of HCC [68-75]. Multiple miRNAs have been reported to be involved in adriamycin resistance in HCC (Table 2).

Several oncogenic miRNAs can promote adriamycin resistance. Let-7a has been found to increase resistance of HepG2 cells to adriamycin [76]. Exogenous expression of miR-519d has also been found to increase resistance of HCC cells to adriamycin by targeting multiple tumor suppressor genes, including $p 21$ and PTEN [77].

By contrast, it has been found that many tumor suppressor miRNAs can reverse adriamycin resistance in HCC. MiR-26a/b, for instance, has been found to be significantly

Table 2 MiRNAs and adriamycin resistance in HCC

\begin{tabular}{|c|c|c|c|}
\hline MiRNAs & Expression $^{1}$ & Pathway & Reference \\
\hline Let-7a & up-regulated & Caspase-3 & {$[76]$} \\
\hline miR-519d & up-regulated & $\begin{array}{l}\text { CDKN1A/p21, } \\
\text { PTEN, AKT3, } \\
\text { TIMP2 }\end{array}$ & {$[77]$} \\
\hline miR-26a/b & down-regulated & ULK1 & {$[78]$} \\
\hline miR-26b & down-regulated & TAK1, TAB3 & {$[79]$} \\
\hline miR-520b & down-regulated & ATG7 & {$[80]$} \\
\hline miR-491-3p & down-regulated & Sp3/ABCB1 & {$[81]$} \\
\hline \multirow[t]{3}{*}{ miR-122 } & down-regulated & MDR1 & [82] \\
\hline & & $\mathrm{ABCB} 1 ; \mathrm{ABCF} 2$ & [83] \\
\hline & & PKM2 & {$[84]$} \\
\hline miR-31 & down-regulated & NDRG3 & {$[85]$} \\
\hline miR-223 & down-regulated & $A B C B 1$ & {$[86]$} \\
\hline miR-133a,miR-326 & down-regulated & $\mathrm{ABCC} 1$ & [87] \\
\hline \multirow[t]{2}{*}{ miR-101 } & down-regulated & $\mathrm{EZH} 2$ & [88] \\
\hline & & Mcl-1 & {$[89]$} \\
\hline miR-199a-3p & down-regulated & mTOR, c-Met & {$[90]$} \\
\hline miR-215 & down-regulated & DHFR, TS & {$[91]$} \\
\hline miR-145 & down-regulated & Smad3 & [92] \\
\hline miR-503 & down-regulated & $\begin{array}{l}\text { MDR1, MRP, } \\
\text { ERCC1, BCl-2 }\end{array}$ & [93] \\
\hline
\end{tabular}

${ }^{1}$ miRNAs either up-regulated or down-regulated in adriamycin resistant hepatocellular carcinoma cells

Note: This table shows 17 miRNAs, their expression level and potential targets in adriamycin resistance of hepatocellular carcinoma 
down-regulated in $30 \mathrm{HCC}$ tissues compared to normal tissues. In addition, exogenous miR-26a/b expression has been found to promote adriamycin sensitivity of HCC cells by targeting ULK1 expression as well as the autophagy signaling pathway in vivo and in vitro [78]. Moreover, miR26b can also sensitize HCC cells to adriamycin through silencing the TAK1 and TAB3 pathways [79]. Similarly, miR-520b has been found to increase the sensitivity of BEL-7402/ADM HCC cells to adriamycin via silencing expression of ATG7, a key autophagy regulator [80]. In HCC cells, expression of miR-491-3p are inversely associated with expression of $A B C B 1$ or $S p 3$. Consistently, ectopic expression of miR-491-3p could sensitize HCC cells to adriamycin by silencing expression of $A B C B 1$ or $S p 3$ [81]. MiR-122, a highly expressed liver-specific miRNA in normal liver tissue, was significantly down-regulated in HCC. The tumor suppressor miR-122 has been found to promote adriamycin sensitivity in HCC cells through inhibiting cell cycle, anti-apoptotic effector factors and $\mathrm{ABC}$ transporters [82-84]. Similarly, miR-31 has been found to increase the sensitivity of HCC cells to adriamycin via silencing the expression of NDRG3 [85]. In addition, down-regulated expression of tumor suppressor miRNAs, such as miR-223, miR-133a, miR-326, miR-101, miR-199a-3p, miR-215, miR145 and miR-503, are significantly correlated with adriamycin resistance of HCC through inhibiting expression of the MDR-related genes. On the contrary, restoring the expression of these miRNAs could reverse adriamycin resistance of HCC cells [86-93].

\section{MiRNAs and resistance to $5-\mathrm{FU}$}

5-FU, a heterocyclic aromatic chemotherapeutic agent, is a broadly used chemotherapeutic drug for HCC treatment. Through inhibiting thymidylate synthase (TS), 5-FU can interfere DNA replication and, thus, result in cell cycle arrest and apoptosis in response to DNA damage [94, 95]. Multiple oncogenic or tumor suppressive miRNAs have been found to be involved in 5-FU resistance (Table 3).

It has been found that several oncogenic miRNAs can promote 5-FU resistance in HCC cells, including miR200a-3p, miR-183, miR-141 and miR-193a-3p. For example, exogenous expression of miR-200a-3p enhanced 5-FU resistance of HCC cells by silencing dual specificity phosphatase 6 (DUSP6) [96]. There was significantly higher miR-183 expression in 5-FU resistant HCC cells compared to 5-FU sensitive HCC cells. Knockdown of oncogenic miR-183 might significantly reverse the 5 -FU tolerance [97]. It has been reported that miR-141 can promote the resistance to 5-FU in HCC cells by inhibiting kelche-like ECH 1-related protein 1 (Keap1) and activating the antioxidant pathway [98]. Moreover, miR193a-3p has been shown to enhance 5-FU resistance of HCC cells via suppressing serine/arginine-rich splicing factor 2 (SRSF2) [99].
Table 3 MiRNAs and 5-FU resistance in HCC

\begin{tabular}{llll}
\hline MiRNAs & Expression $^{1}$ & Pathway & Reference \\
\hline miR-200a-3p & up-regulated & DUSP6 & {$[96]$} \\
miR-183 & up-regulated & IDH2/SOCS6-HIF-1a & {$[97]$} \\
miR-141 & up-regulated & Keap1 & {$[98]$} \\
miR-193a-3p & up-regulated & SRSF2 & {$[99]$} \\
miR-195 & down-regulated & BCl-w & {$[100]$} \\
miR-125b & down-regulated & Hexokinase II & {$[101]$} \\
Let-7 g & down-regulated & HMGA2 & {$[102]$} \\
miR-133a, miR-326 & down-regulated & Bcl-xl & {$[103]$} \\
miR-503 & down-regulated & EIF4E & {$[104]$} \\
\hline
\end{tabular}

${ }^{1}$ miRNAs either up-regulated or down-regulated in resistant hepatocellular carcinoma cells

Note: This table shows 10 miRNAs, their expression level and potential targets in 5-FU resistance of hepatocellular carcinoma

On the contrary, several tumor suppressor miRNAs may be associated with 5-FU resistance. Accumulated evidences indicate that upregulated $B C L-2$ expression confers 5-FU resistance in HCC. It has been found that miR-195 could inhibit expression of $B C L-2$, and an increased sensitivity of drug-resistant $\mathrm{HCC}$ cells to 5-FU has been observed after exogenous overexpression of miR-195 [100]. Ectopic expression of miR-125b has been found to lead to reduced hexokinase 2 (HK2) protein expression and to sensitize HCC cells to 5-FU by inhibiting glycolysis [101]. 5-FU resistant HCC cells have been found to exhibit reduced expression levels of let-7 $\mathrm{g}$ and exogenous expression of let-7g could significantly sensitize HCC cells to 5-FU [102]. Similarly, miR-133a and miR-326 may restore chemosensitivity of HCC to 5FU by targeting $B c l-x l$ [103]. MiR-503, which has been found to be significantly down-regulated in HCC tissues, could lead to significantly increased 5-FU toxicity of HCC cells by suppressing eukaryotic translation initiation factor 4E (EIF4E) [104].

\section{MiRNAs and resistance to cisplatin}

Cisplatin, the first-generation of the platinum chemotherapeutic drugs, can inhibit DNA replication and transcription by forming crosslinks between DNA double strands and exhibits broad-spectrum antitumor activity. Cisplatin is one of the most commonly used chemotherapeutic agents to treat advanced HCC. Several miRNAs have been reported to be involved in cisplatin resistance in HCC (Table 4).

Multiple oncogenic miRNAs can promote cisplatin resistance, such as miR-130a and miR-182. Significantly elevated expression of miR-130a and miR-182 has been observed in tumor tissues from HCC patients and cisplatin-resistant Huh7 and HepG2 cells $[105,106]$. Exogenous expression of miR-130a led to cisplatin tolerance of Huh7 cells by targeting the tumor suppressor 
Table 4 MiRNAs and cisplatin resistance in HCC

\begin{tabular}{llll}
\hline MiRNA & Expression $^{1}$ & Pathway & Reference \\
\hline miR-130a & up-regulated & RUNX3 & {$[105]$} \\
miR-182 & up-regulated & TP53INP1 & {$[106]$} \\
miR-340 & down-regulated & Nrf2 & {$[107]$} \\
miR-363 & down-regulated & Mcl-1 & {$[108]$} \\
\hline
\end{tabular}

${ }^{1}$ miRNAs either up-regulated or down-regulated in cisplatin resistant hepatocellular carcinoma cells

Note: This table shows 4 miRNAs, their expression level and potential targets in cisplatin resistance of hepatocellular carcinoma

RUNX3 and activating the Wnt/ $\beta$-catenin pathway [105]. Similarly, inhibition of miR-182 may partially conquer cisplatin resistance in cisplatin-resistant HepG2 cells by inhibiting tumor suppressor tumor protein 53-induced nucleoprotein 1 (TP53INP1) [106].

By contrast, multiple tumor suppressor miRNAs can reverse cisplatin resistance of $\mathrm{HCC}$. MiR-340, for instance, has been found to be significantly downregulated in cisplatin-resistant $\mathrm{HCC}$ cells. In addition, exogenous miR-340 expression has been found to sensitize chemo-resistant HepG2/CDDP cells to cisplatin through silencing Nrf2 expression, as well as the antioxidant pathway [107]. Additionally, miR-363 has been found to be significantly down-regulated in cisplatin resistant HepG2 cells compared to parental cells, and exogenous miR-363 could significantly reverse cisplatin tolerance in HepG2 cells by targeting $\mathrm{Mcl}-1$ [108].

\section{MiRNAs and resistance to other drugs}

Paclitaxel, cetuximab and etoposide are also commonly used to treat HCC [109-111]. As shown in Table 5, several miRNAs are involved in resistance to these drugs. The tumor suppressor miR-16 has been found to be down-regulated in $\mathrm{HCC}$ tissues and to sensitize $\mathrm{HCC}$ cells to paclitaxel by suppressing the expression of $I K B K B$ as well as the $\mathrm{NF}-\mathrm{kB}$ signaling in vitro and in vivo [35]. Similarly, tumor suppressor miR-9 and miR-23a could sensitize HCC to cetuximab and etoposide by inhibiting expression of eukaryotic translation initiation factor $5 A 2($ eIF-5A-2) and topoisomerase 1 (TOP1), respectively $[112,113]$. As a potential antitumor protein, tumor necrosis factor-related apoptosis inducing ligand (TRAIL) could selectively eliminate various types of HCC cells without exerting toxic effects in normal tissues. MiR-26b and miR-138 have been found to be involved in TRAIL-induced apoptosis and anti-malignancy in HCC. MiR-26b could promote the cytotoxicity of TRAIL in HCC cells by inhibiting $\mathrm{Mcl}-1$ [114]. Similarly, miR-138 was significantly down-regulated in TRAIL resistant HCC cells compared to TRAIL sensitive HCC cells. Ectopic expression of miR-138 has been found to improve the sensitivity of HCC cells to TRAIL [115].
Table 5 MiRNAs and other drugs resistance in HCC

\begin{tabular}{|c|c|c|c|c|}
\hline MiRNAs & Expression $^{1}$ & Pathway & Drug & Reference \\
\hline miR-16 & down-regulated & IKBKB & paclitaxel & {$[35]$} \\
\hline miR-9 & down-regulated & elF-5A-2 & cetuximab & [112] \\
\hline miR-23a & down-regulated & $\begin{array}{l}\text { Topoisomerase } \\
1(\text { TOP1) }\end{array}$ & etoposide & [113] \\
\hline miR-26b & down-regulated & Mcl-1 & TRAIL & [114] \\
\hline miR-138 & down-regulated & $\begin{array}{l}\text { interferon } \\
\text { stimulating } \\
\text { gene } 15\end{array}$ & TRAIL & {$[115]$} \\
\hline miR-93 & up-regulated & PTEN; CDKN1A & tivantinib & {$[50]$} \\
\hline
\end{tabular}

${ }^{1}$ miRNAs either up-regulated or down-regulated in other chemo-drugs resistant hepatocellular carcinoma cells

Note: This table shows 6 miRNAs, their expression level and potential targets in chemoresistance of hepatocellular carcinoma

In addition, miRNAs are also involved in resistance to tivantinib and regorafenib, two novel targeted drugs. Tivantinib, a small, selective oral inhibitor of c-Met receptor tyrosine kinase, provides an option as a secondline treatment for advanced HCC patients who have failed or are intolerant to sorafenib. It has been found that oncogenic miR-93 could confer tivantinib resistance by targeting PTEN and CDKN1A [50]. Interestingly, it has been reported that nine plasma miRNAs, including upregulated plasma expression of miR-30a, miR-122, miR-125b, miR-200a, and miR-374b, diminished levels of miR-15b, miR-107, and miR-320b, as well as the absence of miR-645, can predict regorafenib survival of advanced HCC patients [116].

\section{LncRNAs and therapy resistance}

LncRNAs are a new class of ncRNAs longer than $200 \mathrm{nt}$ and have no protein coding potential. Based on the positions and characteristics in human genome, lncRNAs can be divided into five categories: sense, antisense, bidirectional, intronic and intergenic lncRNAs [117-120]. Multiple lncRNAs are aberrantly expressed in HCC and are significantly associated with metastasis, recurrence, prognosis and chemoresistance through various mechanisms, including interactions with DNA, RNA or proteins to form complexes that regulate the expression of target genes [118, 121-130]. Several lncRNAs have been found to be involved in drug resistance in HCC (Table 6).

It has been reported that lncRNA NR2F1-AS1 is significantly up-regulated in oxaliplatin-resistant HCC tissues and cell lines and confer $\mathrm{HCC}$ resistance to oxaliplatin. NR2F1-AS1 can promote tumor progression in vitro and in vivo. $\mathrm{ABCC} 1$ protein is a member of the superfamily of ATP-binding cassette $(\mathrm{ABC})$ transporters which is involved in multi-drug resistance. In HCC cells, lncRNA NR2F1-AS1 could induce $A B C C 1$ expression via endogenous sponging miR-363 and, thus, antagonize chemosensitivity to oxaliplatin [131]. 
Table 6 LnCRNAs and drug resistance in HCC

\begin{tabular}{lllll}
\hline LnCRNAs & Expression $^{1}$ & Pathway & Drugs & Reference \\
\hline NR2F1-AS1 & up-regulated & miR-363-ABCC1 & oxaliplatin & [131] \\
HANR & up-regulated & GSKIP/P-GSK3 $\beta$ & adriamycin & [132] \\
InCARSR & up-regulated & PTEN-PI3K/Akt & adriamycin & [134] \\
HULC & up-regulated & USP22/Sirt1/ autophagy & oxaliplatin;5-FU; pirarubicin & [135] \\
MALAT1 & up-regulated & miR-216b/ autophagy & 5-FU; adriamycin;mitomycin & [136] \\
TUC338 & up-regulated & RASAL1 & sorafenib & [137] \\
VLDLR & up-regulated & ABCG2 & sorafenib & [138] \\
\hline
\end{tabular}

${ }^{1}$ IncRNAs either up-regulated or down-regulated in chemo-resistant hepatocellular carcinoma cells

Note: This table shows 7 IncRNAs, their expression level and underlying pathways in the chemoresistance of hepatocellular carcinoma

LncRNA HANR (HCC associated long non-coding RNA) shows evidently increased expression in HCC tissues and is associated with poor prognosis of HCC patients. Silencing of lncRNA HANR suppresses HCC proliferation in vitro and in vivo, enhances apoptosis and promotes sensitivity to doxorubicin. It has been found lncRNA HANR could bind to GSKIP and suppress the phosphorylation of GSK3 $\beta$. In hepatocarcinogenesis, suppression of GSK3 $\beta$ phosphorylation and enhanced GSK3 $\beta$ total protein expression regulate glycogen metabolism and cell growth. As a result, alteration of endogenous cellular HANR expression influenced the sensitivity of HCC to doxorubicin-mediated chemotherapy [132].

LncARSR (lncRNA activated in renal cell carcinoma with sunitinib resistance) was firstly reported to be correlated with clinically poor sunitinib response in renal cell carcinoma. It has been found that lncARSR promotes sunitinib resistance via competitively binding $\mathrm{miR}-34 / \mathrm{miR}-$ 449 to facilitate AXL and c-MET expression in renal cell carcinoma cells [133]. Recently, LncARSR is also found to play a part in doxorubicin resistance of HCC. LncARSR is significantly upregulated in HCC, associates with large tumor size and advanced disease stage. Overexpression of lncARSR promotes doxorubicin resistance of HCC cells in vitro and in vivo. In HCC cells, lncARSR physically associates with PTEN mRNA, enhances PTEN mRNA degradation, reduces PTEN expression, and activates the PI3K/Akt signaling pathway [134].

It has been found that lncRNA HULC (highly upregulated lncRNA in $\mathrm{HCC}$ ) could induce autophagy through silencing expression of silent information regulator 1 (Sirt1) protein and weaken the chemosensitivity of oxaliplatin, 5-FU and pirarubicin (THP) in HCC cells. It was also indicated that lncRNA HULC could up-regulate expression of ubiquitin-specific peptidase 22 (USP22) and reduce the ubiquitin-mediated degradation of Sirt1 protein by removing the conjugated polyubiquitin chain of Sirt1 [135]. In addition, lncRNA MALAT1 has been found to be dramatically increased in 5-FU resistant HCC cells of BEL-7402/5-FU and can modulate MDR through impacting autophagy. Knockdown of MALAT1 can reverse 5-FU, adriamycin and mitomycin resistance, diminish LC3-II level and potentiate 5-FU induced apoptosis, which are similar with the effects of autophagy inhibitor of 3-Methyladenine (3-MA) [136].

Therapy resistance to targeted therapy for HCC, i.e. sorafenib, is one of major hurdles in clinics. LncRNA TUC338, for instance, has been found to be involved in the HCC sorafenib resistance. Elevated levels of lncRNA TUC338 were found both in HCC tissues and cell lines. Knockdown of lncRNA TUC338 sensitized HCC cells to sorafenib, which was accompanied by increased expression of RASAL1 [137]. Similarly, lncRNA VLDLR is also involved in sorafenib resistance of HCC cells. VLDLR is one of lncRNAs contained within extracellular vesicles (EV) during chemotherapeutic stress in human HCC. Silencing VLDLR led to the decreased cell viability and altered cell cycle distribution, accompanied with the reduced expression of drug-resistant protein ABCG2 (ATP-binding cassette, subfamily $G$ member 2 ). Overexpression of ABCG2 protein reversed the effects of VLDLR knockdown on sorafenib-induced cell death [138].

There are several novel lncRNAs have been indicated to play crucial functions in $\mathrm{HCC}$ oxaliplatin resistance. Yin et al. evaluated the difference of genome-wide lncRNA expression profiling between oxaliplatin-sensitive and oxaliplatin-resistant HCC cells. A total of 120 differentially expressed lncRNAs were identified, among which 61 lncRNAs were up-regulated and 59 lncRNAs were down-regulated (fold changes $>2$, $P<0.05)$. It was found that ENST00000502804, NR 073453 and ENST00000438347 were also up-regulated in drug-resistant HCC tissues and high expression of ENST00000438347 and ENST00000518376 was associated with bad outcomes of HCC patients [139].

\section{Conclusions and perspectives}

More and more ncRNAs have been identified to be associated with chemoresistance in HCC. The mechanisms underlying the role of ncRNAs in HCC drug 


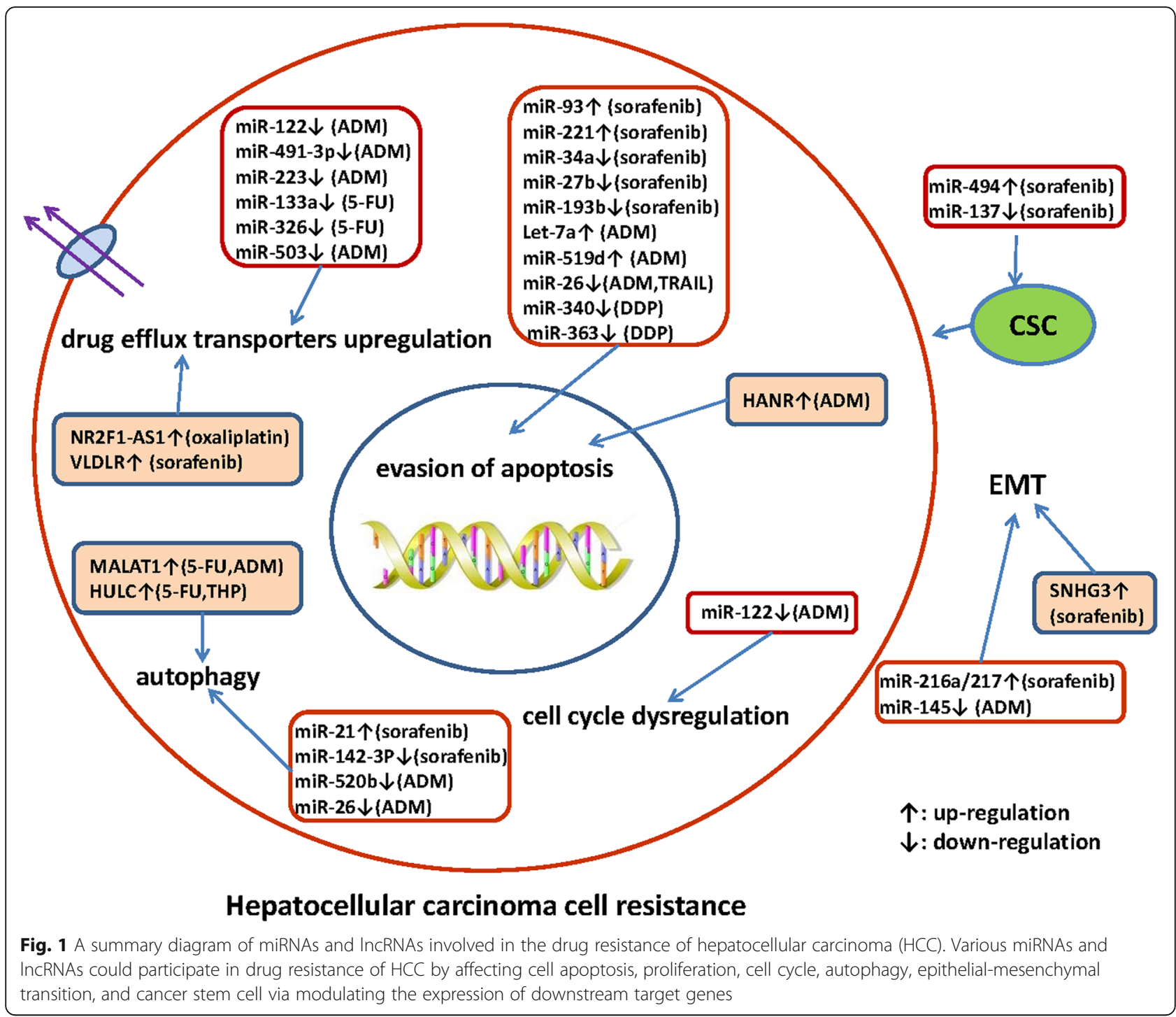

resistance are summarized in Fig. 1. Targeting these dysregulated endogenous miRNAs and/or lncRNAs may be a promising way to reverse drug resistance. For example, nanoparticle delivery of synthetic oligonucleotides targeting oncogenic miRNAs or synthetic tumor suppressor miRNAs or administration with natural agents for the regulation of miRNAs have been investigated as a proof-of-concept for HCC treatment. For IncRNAs, it is applicable that the direct delivery of tumor suppressive lncRNAs molecules to the target cells or the knockdown of oncogenic lncRNAs through specific siRNAs or shRNAs against them. Targeting ncRNAs in combination with conventional chemotherapeutics against $\mathrm{HCC}$ may be a promising alternative for reversing drug resistance and contributing to a better outcome in advanced HCC patients. However, it remains challenging to select critical target ncRNAs from numerous candidates. In order to promote ncRNAbased therapeutic interventions that benefit HCC patients, further investigations on additional translation research and clinical trials are urgently needed, which may ultimately open up potential approaches for overcoming HCC drug resistance.

\section{Abbreviations}

3'-UTR: 3'-untranslated region; 3-MA: 3-Methyladenine; 5-FU: 5-fluorouracil; ABCG2: ATP-binding cassette, subfamily $G$ member 2;

ADAM10: Depolymerization and metalloproteinase domain-containing protein 10; ANT2: Adenine nucleotide transporter 2; AR: Androgen receptor; ATG16L1: Autophagy related 16-like 1; ATG5: Autophagy related 5; CDKN1A: Cell cycle protein-dependent kinase 1A; CIC: Cancer initiation cell; circRNA: circular RNA; CSC: Cancer stem cells; DUSP6: Dual specificity phosphatase 6; EIF4E: Eukaryotic translation initiation factor 4E; elF-5A2: Eukaryotic translation initiation factor 5A2; EMT: Epithelial-mesenchymal transition; GALNT10: Peptide N-acetyl N-acetylgalactosaminyl transferase 10; HCC: Hepatocellular carcinoma; HIF-1a: Hypoxia inducing factor 1a; HK2: Hexokinase 2; IGF-1R: Insulin-like growth factor 1 receptor; 
Keap1: Kelche-like ECH 1-related protein 1; IncRNA: Long noncoding RNA; MDR: Multi-drug resistance; miRNA/miR: microRNA; mRNA: messenger RNA; ncRNA: noncoding RNA; nt: nucleotide; PDK4: Pyruvate dehydrogenase kinase 4; piRNA: PIWI-interacting RNA; RASSF1: Ras association domain family member 1; rRNA: Ribosomal RNA; shRNA: Short hairpin RNA; siRNA: Small interfering RNA; Sirt1: Silent information regulator 1; SLC7A1: Solute carrier family 7 member 1; snoRNA: Small nucleolar RNA; SRF: Serum response factor; SRSF2: Serine/arginine-rich splicing factor 2; THP: Pirarubicin; TOP1: Topoisomerase 1; TP53INP1: Tumor protein 53 -induced nucleoprotein 1; TRAIL: Tumor necrosis factor-related apoptosis inducing ligand; tRNA: Transfer RNA; TS: Thymidylate synthase; USP22: Ubiquitin-specific peptidase 22

\section{Acknowledgements}

Not applicable.

\section{Author's contribution}

LW and MY conceived the review, acquired data, provided project funding and drafted the manuscript. XW, LL, JL, HX, YS, MX and TL undertook the initial research. NZ was involved in writing and reviewing the manuscript. All authors read and approved the final manuscript.

\section{Funding}

This study was financially supported by Science and Technology Development Project of Shandong Province (2016GSF201211) and Shandong Health Department (2016WS0554); National Natural Science Foundation of China (31671300, 31871306); Taishan Scholars Program of Shandong Province (tsqn20161060). The funding bodies did not play any role in writing the manuscript.

\section{Availability of data and materials \\ Not applicable.}

\section{Ethics approval and consent to participate}

Not applicable.

\section{Consent for publication}

Not applicable.

\section{Competing interests}

The authors declare that they have no competing interests.

\section{Author details}

'Shandong Provincial Key Laboratory of Radiation Oncology, Cancer Research Center, Shandong Cancer Hospital and Institute, Shandong First Medical University and Shandong Academy of Medical Sciences, Jinan 250117, Shandong Province, China. ${ }^{2}$ Department of Radiation Oncology, Shandong Cancer Hospital and Institute, Shandong First Medical University and Shandong Academy of Medical Sciences, Jinan 250117, Shandong Province, China. ${ }^{3}$ Department of Intervention Surgery, Shandong Cancer Hospital and Institute, Shandong First Medical University and Shandong Academy of Medical Sciences, Jinan, China.

\section{Received: 13 August 2019 Accepted: 4 October 2019}

\section{Published online: 25 October 2019}

\section{References}

1. El-Serag HB. Hepatocellular carcinoma. N Engl J Med. 2011;365(12):1118-27.

2. Shen YC, Lin ZZ, Hsu CH, Hsu C, Shao YY, Cheng AL. Clinical trials in hepatocellular carcinoma: an update. Liver Cancer. 2013;2(3-4):345-64.

3. Grem JL. 5-fluorouracil: forty-plus and still ticking. A review of its preclinical and clinical development. Investig New Drugs. 2000;18(4):299-313.

4. Kalyan A, Nimeiri H, Kulik L. Systemic therapy of hepatocellular carcinoma: current and promising. Clin Liver Dis. 2015;19(2):421-32.

5. Llovet JM, Villanueva A, Lachenmayer A, Finn RS. Advances in targeted therapies for hepatocellular carcinoma in the genomic era. Nat Rev Clin Oncol. 2015;12(7):408-24.

6. Butler EB, Zhao Y, Munoz-Pinedo C, Lu J, Tan M. Stalling the engine of resistance: targeting cancer metabolism to overcome therapeutic resistance. Cancer Res. 2013;73(9):2709-17.
7. Salehan MR, Morse HR. DNA damage repair and tolerance: a role in chemotherapeutic drug resistance. Br J Biomed Sci. 2013;70(1):31-40.

8. Xing F, Wang S, Zhou J. The expression of MicroRNA-598 inhibits ovarian Cancer cell proliferation and metastasis by targeting URI. Mol Ther Oncolytics. 2019;12:9-15.

9. Wang WJ, Li HT, Yu JP, Han XP, Xu ZP, Li YM, et al. A competing endogenous RNA network reveals novel potential IncRNA, miRNA, and mRNA biomarkers in the prognosis of human Colon adenocarcinoma. J Surg Res. 2019;235:22-33.

10. Mai D, Ding P, Tan L, Zhang J, Pan Z, Bai R, et al. PIWl-interacting RNA54265 is oncogenic and a potential therapeutic target in colorectal adenocarcinoma. Theranostics. 2018;8(19):5213-30.

11. Vychytilova-Faltejskova P, Stitkovcova K, Radova L, Sachlova M, Kosarova Z, Slaba K, et al. Circulating PIWI-interacting RNAs piR-5937 and piR-28876 are promising diagnostic biomarkers of Colon Cancer. Cancer Epidemiol Biomark Prev. 2018;27(9):1019-28.

12. Yi C, Wan X, Zhang Y, Fu F, Zhao C, Qin R, et al. SNORA42 enhances prostate cancer cell viability, migration and EMT and is correlated with prostate cancer poor prognosis. Int J Biochem Cell Biol. 2018;102:138-50.

13. Wang H, Ma P, Liu P, Chen B, Liu Z. Small nucleolar RNA U2_19 promotes hepatocellular carcinoma progression by regulating Wnt/beta-catenin signaling. Biochem Biophys Res Commun. 2018;500(2):351-6.

14. Wu L, Zheng J, Chen P, Liu Q, Yuan Y. Small nucleolar RNA ACA11 promotes proliferation, migration and invasion in hepatocellular carcinoma by targeting the PIJKAKT signaling pathway. Biomed Pharmacother. 2017;90:705-12.

15. Okugawa Y, Toiyama Y, Toden S, Mitoma H, Nagasaka T, Tanaka K, et al. Clinical significance of SNORA42 as an oncogene and a prognostic biomarker in colorectal cancer. Gut. 2017;66(1):107-17.

16. Qin XG, Zeng JH, Lin P, Mo WJ, Li Q, Feng ZB, et al. Prognostic value of small nuclear RNAs (snRNAs) for digestive tract pan- adenocarcinomas identified by RNA sequencing data. Pathol Res Pract. 2019;215(3):414-26.

17. Cech TR, Steitz JA. The noncoding RNA revolution-trashing old rules to forge new ones. Cell. 2014;157(1):77-94.

18. Dhamija S, Diederichs S. From junk to master regulators of invasion: IncRNA functions in migration. EMT and metastasis Int J Cancer. 2016; 139(2):269-80.

19. Yang F, Jiang Y, LV LZ. Long non-coding RNA XLOC_010235 correlates with poor prognosis and promotes tumorigenesis of hepatocellular carcinoma. Eur Rev Med Pharmacol Sci. 2017;21(21):4867-74.

20. Tan HY, Zheng YB, Liu J. Serum miR-199a as a potential diagnostic biomarker for detection of colorectal cancer. Eur Rev Med Pharmacol Sci. 2018;22(24):8657-63.

21. Wang J, Yang K, Yuan W, Gao Z. Determination of serum Exosomal H19 as a noninvasive biomarker for bladder Cancer diagnosis and prognosis. Med Sci Monit. 2018;24:9307-16.

22. Hu D, Zhan Y, Zhu K, Bai M, Han J, Si Y, et al. Plasma Exosomal Long noncoding RNAs serve as biomarkers for early detection of colorectal Cancer. Cell Physiol Biochem. 2018;51(6):2704-15.

23. Gao H, Hao G, Sun Y, Li L, Wang Y. Long noncoding RNA H19 mediated the chemosensitivity of breast cancer cells via Wnt pathway and EMT process. Onco Targets Ther. 2018;11:8001-12.

24. Zeng L, Liao Q, Zou Z, Wen Y, Wang J, Liu C, et al. Long non-coding RNA XLOC_006753 promotes the development of multidrug resistance in gastric Cancer cells through the PI3K/AKT/mTOR signaling pathway. Cell Physiol Biochem. 2018;51(3):1221-36.

25. Que KT, Zhou Y, You Y, Zhang Z, Zhao XP, Gong JP, et al. MicroRNA-31-5p regulates chemosensitivity by preventing the nuclear location of PARP1 in hepatocellular carcinoma. J Exp Clin Cancer Res. 2018;37(1):268.

26. Chen M, Wu L, Tu J, Zhao Z, Fan X, Mao J, et al. miR-590-5p suppresses hepatocellular carcinoma chemoresistance by targeting YAP1 expression. EBioMedicine. 2018;35:142-54.

27. Yuan G, Wu H, Du Y, He F. Tumor suppressor role of microRNA-545 in oral squamous cell carcinoma. Oncol Lett. 2019;17(2):2063-8.

28. Chen L, Gao H, Liang J, Qiao J, Duan J, Shi H, et al. miR-203a-3p promotes colorectal cancer proliferation and migration by targeting PDE4D. Am J Cancer Res. 2018;8(12):2387-401.

29. Xu B, Lu X, Zhao Y, Liu C, Huang X, Chen S, et al. MicroRNA-135a induces prostate cancer cell apoptosis via inhibition of STAT6. Oncol Lett. 2019;17(2):1889-95.

30. Ni JS, Zheng H, Huang ZP, Hong YG, Ou YL, Tao YP, et al. MicroRNA-197-3p acts as a prognostic marker and inhibits cell invasion in hepatocellular carcinoma. Oncol Lett. 2019;17(2):2317-27. 
31. Zhou Y, Ren H, Dai B, Li J, Shang L, Huang J, et al. Hepatocellular carcinoma-derived exosomal miRNA-21 contributes to tumor progression by converting hepatocyte stellate cells to cancer-associated fibroblasts. J Exp Clin Cancer Res. 2018;37(1):324.

32. Wang Y, Chang W, Chang W, Chang X, Zhai S, Pan G, et al. MicroRNA-376c$3 p$ facilitates human hepatocellular carcinoma progression via repressing AT-rich interaction domain 2. J Cancer. 2018;9(22):4187-96.

33. Jiang XM, Yu XN, Liu TT, Zhu HR, Shi X, Bilegsaikhan E, et al. microRNA19a-3p promotes tumor metastasis and chemoresistance through the PTEN/Akt pathway in hepatocellular carcinoma. Biomed Pharmacother. 2018;105:1147-54

34. Tian T, Fu X, Lu J, Ruan Z, Nan K, Yao Y, et al. MicroRNA-760 inhibits doxorubicin resistance in hepatocellular carcinoma through regulating Notch1/Hes1-PTEN/Akt signaling pathway. J Biochem Mol Toxicol. 2018; 32(8):e22167.

35. Huang Y, Chen G, Wang Y, He R, Du J, Jiao X, et al. Inhibition of microRNA16 facilitates the paclitaxel resistance by targeting IKBKB via NF-kappaB signaling pathway in hepatocellular carcinoma. Biochem Biophys Res Commun. 2018;503(2):1035-41.

36. Zhang K, Chen J, Zhou H, Chen Y, Zhi Y, Zhang B, et al. PU.1/microRNA-142$3 p$ targets ATG5/ATG16L1 to inactivate autophagy and sensitize hepatocellular carcinoma cells to sorafenib. Cell Death Dis. 2018;9(3):312.

37. Zhang G, Li N, Li Z, Zhu Q, Li F, Yang C, et al. microRNA-4717 differentially interacts with its polymorphic target in the PD1 3' untranslated region: A mechanism for regulating PD-1 expression and function in HBV-associated liver diseases. Oncotarget. 2015;6(22):18933-44.

38. Wilhelm SM, Carter C, Tang L, Wilkie D, McNabola A, Rong H, et al. BAY 439006 exhibits broad spectrum oral antitumor activity and targets the RAF/ MEKJERK pathway and receptor tyrosine kinases involved in tumor progression and angiogenesis. Cancer Res. 2004;64(19):7099-109.

39. Yu C, Bruzek LM, Meng XW, Gores GJ, Carter CA, Kaufmann SH, et al. The role of $\mathrm{MCl}-1$ downregulation in the proapoptotic activity of the multikinase inhibitor BAY 43-9006. Oncogene. 2005;24(46):6861-9.

40. Sonntag R, Gassler N, Bangen JM, Trautwein C, Liedtke C. Pro-apoptotic Sorafenib signaling in murine hepatocytes depends on malignancy and is associated with PUMA expression in vitro and in vivo. Cell Death Dis. 2014;5:e1030.

41. Wang Z, Hu P, Tang F, Xie C. HDAC6-mediated EGFR stabilization and activation restrict cell response to sorafenib in non-small cell lung cancer cells. Med Oncol. 2016;33(5):50.

42. Xiang QF, Zhan MX, Li Y, Liang H, Hu C, Huang YM, et al. Activation of MET promotes resistance to sorafenib in hepatocellular carcinoma cells via the AKT/ERK1/2-EGR1 pathway. Artif Cells Nanomed Biotechnol. 2019;47(1):83-9.

43. Xu H, Zhao L, Fang Q, Sun J, Zhang S, Zhan C, et al. MiR-338-3p inhibits hepatocarcinoma cells and sensitizes these cells to sorafenib by targeting hypoxia-induced factor 1alpha. PLoS One. 2014;9(12):e115565.

44. Liu H, Wang M, Liang N, Guan L. PDCD2 sensitizes HepG2 cells to sorafenib by suppressing epithelialmesenchymal transition. Mol Med Rep. 2019;19(3):2173-9.

45. Pollutri D, Patrizi C, Marinelli S, Giovannini C, Trombetta E, Giannone FA, et al. The epigenetically regulated miR-494 associates with stem-cell phenotype and induces sorafenib resistance in hepatocellular carcinoma. Cell Death Dis. 2018;9(1):4.

46. Fornari F, Pollutri D, Patrizi C, La Bella T, Marinelli S, Casadei Gardini A, et al. In hepatocellular carcinoma miR-221 modulates Sorafenib resistance through inhibition of Caspase-3-mediated apoptosis. Clin Cancer Res. 2017; 23(14):3953-65.

47. Liu K, Liu S, Zhang W, Ji B, Wang Y, Liu Y. miR222 regulates sorafenib resistance and enhance tumorigenicity in hepatocellular carcinoma. Int J Oncol. 2014;45(4):1537-46.

48. Zhang PF, Wang F, Wu J, Wu Y, Huang W, Liu D, et al. LncRNA SNHG3 induces EMT and sorafenib resistance by modulating the miR-128/CD151 pathway in hepatocellular carcinoma. J Cell Physiol. 2019;234(3):2788-94.

49. He C, Dong X, Zhai B, Jiang X, Dong D, Li B, et al. MiR-21 mediates sorafenib resistance of hepatocellular carcinoma cells by inhibiting autophagy via the PTEN/Akt pathway. Oncotarget. 2015;6(30):28867-81.

50. Ohta K, Hoshino H, Wang J, Ono S, lida Y, Hata K, et al. MicroRNA93 activates c-met/PI3K/Akt pathway activity in hepatocellular carcinoma by directly inhibiting PTEN and CDKN1A. Oncotarget. 2015;6(5):3211-24

51. Xia H, Ooi LL, Hui KM. MicroRNA-216a/217-induced epithelial-mesenchymal transition targets PTEN and SMAD7 to promote drug resistance and recurrence of liver cancer. Hepatology. 2013;58(2):629-41.
52. Azumi J, Tsubota T, Sakabe T, Shiota G. miR-181a induces sorafenib resistance of hepatocellular carcinoma cells through downregulation of RASSF1 expression. Cancer Sci. 2016;107(9):1256-62.

53. Liu K, Liu S, Zhang W, Jia B, Tan L, Jin Z, et al. miR-494 promotes cell proliferation, migration and invasion, and increased sorafenib resistance in hepatocellular carcinoma by targeting PTEN. Oncol Rep. 2015;34(2):1003-10.

54. Xu Y, Huang J, Ma L, Shan J, Shen J, Yang Z, et al. MicroRNA-122 confers sorafenib resistance to hepatocellular carcinoma cells by targeting IGF-1R to regulate RAS/RAF/ERK signaling pathways. Cancer Lett. 2016;371(2):171-81.

55. Bai S, Nasser MW, Wang B, Hsu SH, Datta J, Kutay H, et al. MicroRNA-122 inhibits tumorigenic properties of hepatocellular carcinoma cells and sensitizes these cells to sorafenib. J Biol Chem. 2009;284(46):32015-27.

56. Song K, Kwon H, Han C, Zhang J, Dash S, Lim K, et al. Active glycolytic metabolism in CD133(+) hepatocellular cancer stem cells: regulation by MIR-122. Oncotarget. 2015;6(38):40822-35.

57. Kishikawa T, Otsuka M, Tan PS, Ohno M, Sun X, Yoshikawa T, et al. Decreased miR122 in hepatocellular carcinoma leads to chemoresistance with increased arginine. Oncotarget. 2015;6(10):8339-52.

58. Wu Q, Liu HO, Liu YD, Liu WS, Pan D, Zhang WJ, et al. Decreased expression of hepatocyte nuclear factor 4alpha (Hnf4alpha)/microRNA122 (miR-122) axis in hepatitis B virus-associated hepatocellular carcinoma enhances potential oncogenic GALNT10 protein activity. J Biol Chem. 2015;290(2):1170-85.

59. Yang F, Li QJ, Gong ZB, Zhou L, You N, Wang S, et al. MicroRNA-34a targets $\mathrm{BCl}-2$ and sensitizes human hepatocellular carcinoma cells to sorafenib treatment. Technol Cancer Res Treat. 2014;13(1):77-86.

60. Mu W, Hu C, Zhang H, Qu Z, Cen J, Qiu Z, et al. miR-27b synergizes with anticancer drugs via p53 activation and CYP1B1 suppression. Cell Res. 2015; 25(4):477-95.

61. Shimizu S, Takehara T, Hikita H, Kodama T, Miyagi T, Hosui A, et al. The let-7 family of microRNAs inhibits BCl-xL expression and potentiates sorafenibinduced apoptosis in human hepatocellular carcinoma. J Hepatol. 2010; 52(5):698-704

62. Braconi C, Valeri N, Gasparini P, Huang N, Taccioli C, Nuovo G, et al. Hepatitis $C$ virus proteins modulate microRNA expression and chemosensitivity in malignant hepatocytes. Clin Cancer Res. 2010; 16(3):957-66

63. Mao K, Zhang J, He C, Xu K, Liu J, Sun J, et al. Restoration of miR-193b sensitizes hepatitis B virus-associated hepatocellular carcinoma to sorafenib. Cancer Lett. 2014;352(2):245-52.

64. Sun $H$, Cui C, Xiao F, Wang H, Xu J, Shi X, et al. miR-486 regulates metastasis and chemosensitivity in hepatocellular carcinoma by targeting CLDN10 and CITRON. Hepatol Res. 2015;45(13):1312-22.

65. Xu J, Lin H, Li G, Sun Y, Chen J, Shi L, et al. The miR-367-3p increases Sorafenib chemotherapy efficacy to suppress hepatocellular carcinoma metastasis through altering the androgen receptor signals. EBioMedicine. 2016;12:55-67.

66. Lu AQ, Lv B, Qiu F, Wang XY, Cao XH. Upregulation of miR-137 reverses sorafenib resistance and cancer-initiating cell phenotypes by degrading ANT2 in hepatocellular carcinoma. Oncol Rep. 2017;37(4):2071-8.

67. Kabir TD, Ganda C, Brown RM, Beveridge DJ, Richardson KL, Chaturvedi V, et al. A microRNA-7/growth arrest specific 6/TYRO3 axis regulates the growth and invasiveness of sorafenib-resistant cells in human hepatocellular carcinoma. Hepatology. 2018;67(1):216-31.

68. Liu W, Meng Q, Sun Y, Wang C, Huo X, Liu Z, et al. Targeting P-glycoprotein: Nelfinavir reverses Adriamycin resistance in K562/ADR cells. Cell Physiol Biochem. 2018;51(4):1616-31.

69. Han J, Jun Y, Kim SH, Hoang HH, Jung Y, Kim S, et al. Rapid emergence and mechanisms of resistance by $U 87$ glioblastoma cells to doxorubicin in an in vitro tumor microfluidic ecology. Proc Natl Acad Sci U S A. 2016;113(50): 14283-8.

70. Gao Z, Li Z, Liu Y, Liu Z. Forkhead box O3 promotes colon cancer proliferation and drug resistance by activating MDR1 expression. Mol Genet Genomic Med. 2019:e554.

71. Chen JM, Bai JY, Yang KX. Effect of resveratrol on doxorubicin resistance in breast neoplasm cells by modulating PI3K/Akt signaling pathway. IUBMB Life. 2018;70(6):491-500.

72. Fan Y, Li M, Ma K, Hu Y, Jing J, Shi Y, et al. Dual-target MDM2/MDMX inhibitor increases the sensitization of doxorubicin and inhibits migration and invasion abilities of triple-negative breast cancer cells through activation of TAB1/TAK1/p38 MAPK pathway. Cancer Biol Ther. 2018:1-16. 
73. Shin DH, Choi YJ, Park JW. SIRT1 and AMPK mediate hypoxia-induced resistance of non-small cell lung cancers to cisplatin and doxorubicin. Cancer Res. 2014;74(1):298-308.

74. Mi H, Wang $X$, Wang F, Li L, Zhu M, Wang $N$, et al. miR-381 induces sensitivity of breast cancer cells to doxorubicin by inactivation of MAPK signaling via FYN. Eur J Pharmacol. 2018;839:66-75.

75. Zhou B, Li L, Li Y, Sun H, Zeng C. Long noncoding RNA SNHG12 mediates doxorubicin resistance of osteosarcoma via miR-320a/MCL1 axis. Biomed Pharmacother. 2018;106:850-7.

76. Tsang WP, Kwok TT. Let-7a microRNA suppresses therapeutics-induced cancer cell death by targeting caspase-3. Apoptosis. 2008;13(10):1215-22.

77. Fornari F, Milazzo M, Chieco P, Negrini M, Marasco E, Capranico G, et al. In hepatocellular carcinoma miR-519d is up-regulated by p53 and DNA hypomethylation and targets CDKN1A/p21, PTEN, AKT3 and TIMP2. J Pathol. 2012;227(3):275-85.

78. Jin F, Wang Y, Li M, Zhu Y, Liang H, Wang C, et al. MiR-26 enhances chemosensitivity and promotes apoptosis of hepatocellular carcinoma cells through inhibiting autophagy. Cell Death Dis. 2017;8(1):e2540.

79. Zhao N, Wang R, Zhou L, Zhu Y, Gong J, Zhuang SM. MicroRNA-26b suppresses the NF-kappaB signaling and enhances the chemosensitivity of hepatocellular carcinoma cells by targeting TAK1 and TAB3. Mol Cancer. 2014;13:35.

80. Gao AM, Zhang XY, Hu JN, Ke ZP. Apigenin sensitizes hepatocellular carcinoma cells to doxorubic through regulating miR-520b/ATG7 axis. Chem Biol Interact. 2018;280:45-50.

81. Zhao Y, Qi X, Chen J, Wei W, Yu C, Yan H, et al. The miR-491-3p/Sp3/ABCB1 axis attenuates multidrug resistance of hepatocellular carcinoma. Cancer Lett. 2017;408:102-11.

82. Xu Y, Xia F, Ma L, Shan J, Shen J, Yang Z, et al. MicroRNA-122 sensitizes HCC cancer cells to adriamycin and vincristine through modulating expression of MDR and inducing cell cycle arrest. Cancer Lett. 2011;310(2):160-9.

83. Yahya SMM, Fathy SA, El-Khayat ZA, El-Toukhy SE, Hamed AR, Hegazy MGA, et al. Possible role of microRNA-122 in modulating multidrug resistance of hepatocellular carcinoma. Indian J Clin Biochem. 2018;33(1):21-30.

84. Pan C, Wang X, Shi K, Zheng Y, Li J, Chen Y, et al. MiR-122 reverses the doxorubicin-resistance in hepatocellular carcinoma cells through regulating the tumor metabolism. PLoS One. 2016;11(5):e0152090.

85. Du Z, Niu S, Xu X, Xu Q. MicroRNA31-NDRG3 regulation axes are essential for hepatocellular carcinoma survival and drug resistance. Cancer Biomark. 2017;19(2):221-30.

86. Yang T, Zheng ZM, Li XN, Li ZF, Wang Y, Geng YF, et al. MiR-223 modulates multidrug resistance via downregulation of $A B C B 1$ in hepatocellular carcinoma cells. Exp Biol Med (Maywood). 2013;238(9):1024-32.

87. Ma J, Wang T, Guo R, Yang X, Yin J, Yu J, et al. Involvement of miR-133a and miR-326 in ADM resistance of HepG2 through modulating expression of ABCC1. J Drug Target. 2015;23(6):519-24.

88. Xu L, Beckebaum S, lacob S, Wu G, Kaiser GM, Radtke A, et al. MicroRNA-101 inhibits human hepatocellular carcinoma progression through EZH2 downregulation and increased cytostatic drug sensitivity. J Hepatol. 2014; 60(3):590-8

89. He H, Tian W, Chen H, Deng Y. MicroRNA-101 sensitizes hepatocellular carcinoma cells to doxorubicin-induced apoptosis via targeting Mcl-1. Mol Med Rep. 2016;13(2):1923-9.

90. Fornari F, Milazzo M, Chieco P, Negrini M, Calin GA, Grazi GL, et al. MiR199a-3p regulates $m$ TOR and c-met to influence the doxorubicin sensitivity of human hepatocarcinoma cells. Cancer Res. 2010;70(12):5184-93.

91. Wang L, Wang YM, Xu S, Wang WG, Chen Y, Mao JY, et al. MicroRNA-215 is upregulated by treatment with Adriamycin and leads to the chemoresistance of hepatocellular carcinoma cells and tissues. Mol Med Rep. 2015;12(4):5274-80.

92. Ju BL, Chen YB, Zhang WY, Yu CH, Zhu DQ, Jin J. miR-145 regulates chemoresistance in hepatocellular carcinoma via epithelial mesenchymal transition. Cell Mol Biol (Noisy-le-grand). 2015;61(3):12-6.

93. Wang D, Zhang N, Ye Y, Qian J, Zhu Y, Wang C. Role and mechanisms of microRNA503 in drug resistance reversal in HepG2/ADM human hepatocellular carcinoma cells. Mol Med Rep. 2014;10(6):3268-74.

94. Longley DB, Harkin DP, Johnston PG. 5-fluorouracil: mechanisms of action and clinical strategies. Nat Rev Cancer. 2003;3(5):330-8.

95. Noordhuis P, Holwerda U, Van der Wilt CL, Van Groeningen CJ, Smid K, Meijer $\mathrm{S}$, et al. 5-fluorouracil incorporation into RNA and DNA in relation to thymidylate synthase inhibition of human colorectal cancers. Ann Oncol. 2004;15(7):1025-32.
96. Lee H, Kim C, Kang H, Tak H, Ahn S, Yoon SK, et al. microRNA-200a-3p increases 5-fluorouracil resistance by regulating dual specificity phosphatase 6 expression. Exp Mol Med. 2017;49(5):e327.

97. Wang XJ, Zhang DL, Fu C, Wei BZ, Li GJ. MiR-183 modulates multidrug resistance in hepatocellular cancer (HCC) cells via miR-183-IDH2/ SOCS6-HIF-1alpha feedback loop. Eur Rev Med Pharmacol Sci. 2016; 20(10):2020-7.

98. Shi L, Wu L, Chen Z, Yang J, Chen X, Yu F, et al. MiR-141 activates Nrf2dependent antioxidant pathway via Down-regulating the expression of Keap1 conferring the resistance of hepatocellular carcinoma cells to 5fluorouracil. Cell Physiol Biochem. 2015;35(6):2333-48.

99. Ma K, He Y, Zhang H, Fei Q, Niu D, Wang D, et al. DNA methylationregulated miR-193a-3p dictates resistance of hepatocellular carcinoma to 5 -fluorouracil via repression of SRSF2 expression. J Biol Chem. 2012; 287(8):5639-49.

100. Yang X, Yin J, Yu J, Xiang Q, Liu Y, Tang S, et al. miRNA-195 sensitizes human hepatocellular carcinoma cells to 5-FU by targeting BCL-w. Oncol Rep. 2012;27(1):250-7.

101. Jiang JX, Gao S, Pan YZ, Yu C, Sun CY. Overexpression of microRNA-125b sensitizes human hepatocellular carcinoma cells to 5-fluorouracil through inhibition of glycolysis by targeting hexokinase II. Mol Med Rep. 2014;10(2):995-1002.

102. Tang H, Zhang P, Xiang Q, Yin J, Yu J, Yang X, et al. Let-7 g microRNA sensitizes fluorouracil-resistant human hepatoma cells. Pharmazie. 2014; 69(4):287-92.

103. Ma J, Wang T, Guo R, Yang X, Yin J, Yu J, et al. MicroRNA133a and microRNA326 cocontribute to hepatocellular carcinoma 5fluorouracil and cisplatin sensitivity by directly targeting Bcell lymphomaextra large. Mol Med Rep. 2015;12(4):6235-40.

104. Yang X, Zang J, Pan X, Yin J, Xiang Q, Yu J, et al. miR-503 inhibits proliferation making human hepatocellular carcinoma cells susceptible to 5fluorouracil by targeting EIF4E. Oncol Rep. 2017;37(1):563-70.

105. Xu N, Shen C, Luo Y, Xia L, Xue F, Xia Q, et al. Upregulated miR-130a increases drug resistance by regulating RUNX3 and Wnt signaling in cisplatin-treated HCC cell. Biochem Biophys Res Commun. 2012;425(2):468-72

106. Qin J, Luo M, Qian H, Chen W. Upregulated miR-182 increases drug resistance in cisplatin-treated HCC cell by regulating TP53INP1. Gene. 2014; 538(2):342-7.

107. Shi L, Chen ZG, Wu LL, Zheng JJ, Yang JR, Chen XF, et al. miR-340 reverses cisplatin resistance of hepatocellular carcinoma cell lines by targeting Nrf2dependent antioxidant pathway. Asian Pac J Cancer Prev. 2014;15(23):10439-44.

108. Ou Y, Zhai D, Wu N, Li X. Downregulation of miR-363 increases drug resistance in cisplatin-treated HepG2 by dysregulating Mcl-1. Gene. 2015;572(1):116-22.

109. Chen L, Liu Y, Wang W, Liu K. Effect of integrin receptor-targeted liposomal paclitaxel for hepatocellular carcinoma targeting and therapy. Oncol Lett. 2015;10(1):77-84.

110. Poggi G, Montagna B, Melchiorre F, Quaretti P, Delmonte A, Riccardi A, et al Hepatic intra-arterial cetuximab in combination with 5-fluorouracil and cisplatin as salvage treatment for sorafenib-refractory hepatocellular carcinoma. Anticancer Res. 2011;31(11):3927-33.

111. Dostal Z, Kosina P, Mlejnek P, Kikalova K, Modriansky M. Mifepristone potentiates etoposide toxicity in Hep G2 cells by modulating drug transport. Toxicol in Vitro. 2019;54:33-40.

112. Xue F, Liang Y, Li Z, Liu Y, Zhang H, Wen Y, et al. MicroRNA-9 enhances sensitivity to cetuximab in epithelial phenotype hepatocellular carcinoma cells through regulation of the eukaryotic translation initiation factor $5 \mathrm{~A}-2$. Oncol Lett. 2018;15(1):813-20.

113. Wang N, Zhu M, Tsao SW, Man K, Zhang Z, Feng Y. MiR-23a-mediated inhibition of topoisomerase 1 expression potentiates cell response to etoposide in human hepatocellular carcinoma. Mol Cancer. 2013;12(1):119.

114. Jiang C, Long J, Liu B, Xie X, Kuang M. Mcl-1 is a novel target of miR-26b that is associated with the apoptosis induced by TRAIL in HCC cells. Biomed Res Int. 2015;2015:572738.

115. Zuo C, Sheng X, Liu Z, Ma M, Xiong S, Deng H, et al. MicroRNA-138 enhances TRAIL-induced apoptosis through interferon-stimulated gene 15 downregulation in hepatocellular carcinoma cells. Tumour Biol. 2017;39(6): 1010428317710410 .

116. Teufel M, Seidel H, Kochert K, Meinhardt G, Finn RS, Llovet JM, et al. Biomarkers associated with response to Regorafenib in patients with hepatocellular carcinoma. Gastroenterology. 2019;156(6):1731-41.

117. Mondal T, Juvvuna PK, Kirkeby A, Mitra S, Kosalai ST, Traxler L, et al. Senseantisense IncRNA pair encoded by locus 6 p22.3 determines neuroblastoma 
susceptibility via the USP36-CHD7-SOX9 regulatory Axis. Cancer Cell. 2018; 33(3):417-34 e7.

118. Zhang P, Dong Q, Zhu H, Li S, Shi L, Chen X. Long non-coding antisense RNA GAS6-AS1 supports gastric cancer progression via increasing GAS6 expression. Gene. 2019;696:1-9.

119. Tzadok S, Caspin Y, Hachmo Y, Canaani D, Dotan I. Directionality of noncoding human RNAs: how to avoid artifacts. Anal Biochem. 2013;439(1):23-9.

120. Hamazaki N, Nakashima K, Hayashi K, Imamura T. Detection of bidirectional promoter-derived IncRNAs from small-scale samples using pre-amplificationfree directional RNA-seq method. Methods Mol Biol. 2017;1605:83-103.

121. Shi L, Hong X, Ba L, He X, Xiong Y, Ding Q, et al. Long non-coding RNA ZNFX1-AS1 promotes the tumor progression and metastasis of colorectal cancer by acting AS a competing endogenous RNA of miR-144 to regulate EZH2 expression. Cell Death Dis. 2019;10(3):150.

122. Lou Y, Yu Y, Xu X, Zhou S, Shen H, Fan T, et al. Long non-coding RNA LUCAT1 promotes tumourigenesis by inhibiting ANXA2 phosphorylation in hepatocellular carcinoma. J Cell Mol Med. 2019;23(3):1873-84.

123. Liu D, Gao M, Wu K, Zhu D, Yang Y, Zhao S. LINC00152 facilitates tumorigenesis in esophageal squamous cell carcinoma via miR-153-3p/FYN axis. Biomed Pharmacother. 2019;112:108654.

124. Wang $Y$, Yang $L$, Chen T, Liu X, Guo Y, Zhu Q, et al. A novel IncRNA MCM3AP-AS1 promotes the growth of hepatocellular carcinoma by targeting miR-194-5p/FOXA1 axis. Mol Cancer. 2019;18(1):28.

125. Chen LL, He J, Qiu XT, Yu J, Wang ZM. The prognostic roles of long noncoding RNA SNHG17 in the patients with gastric cancer. Eur Rev Med Pharmacol Sci. 2019;23(3):1063-8.

126. Gao R, Fang C, Xu J, Tan H, Li P, Ma L. LncRNA CACS15 contributes to oxaliplatin resistance in colorectal cancer by positively regulating $\mathrm{ABCC}$ through sponging miR-145. Arch Biochem Biophys. 2019;663:183-91.

127. Shi C, Wang M. LINC01118 modulates paclitaxel resistance of epithelial ovarian Cancer by regulating miR-134/ABCC1. Med Sci Monit. 2018;24:8831-9.

128. Wang L, Shang $X$, Feng Q. LncRNA TATDN1 contributes to the cisplatin resistance of non-small cell lung cancer through TATDN1/miR-451/TRIM66 axis. Cancer Biol Ther. 2019;20(3):261-71.

129. Dong X, Fang Z, Yu M, Zhang L, Xiao R, Li X, et al. Knockdown of Long noncoding RNA HOXA-AS2 suppresses Chemoresistance of acute myeloid leukemia via the miR-520c-3p/S100A4 Axis. Cell Physiol Biochem. 2018;51(2):886-96.

130. Wang M, Han D, Yuan Z, Hu H, Zhao Z, Yang R, et al. Long non-coding RNA H19 confers 5-Fu resistance in colorectal cancer by promoting SIRT1mediated autophagy. Cell Death Dis. 2018;9(12):1149.

131. Huang $\mathrm{H}$, Chen J, Ding CM, Jin X, Jia ZM, Peng J. LncRNA NR2F1-AS1 regulates hepatocellular carcinoma oxaliplatin resistance by targeting ABCC1 via miR-363. J Cell Mol Med. 2018;22(6):3238-45.

132. Xiao J, Lv Y, Jin F, Liu Y, Ma Y, Xiong Y, et al. LncRNA HANR promotes tumorigenesis and increase of Chemoresistance in hepatocellular carcinoma. Cell Physiol Biochem. 2017:43(5):1926-38.

133. Qu L, Ding J, Chen C, Wu ZJ, Liu B, Gao Y, et al. Exosome-transmitted IncARSR promotes Sunitinib resistance in renal Cancer by acting as a competing endogenous RNA. Cancer Cell. 2016;29(5):653-68.

134. Li Y, Ye Y, Feng B, Qi Y. Long noncoding RNA IncARSR promotes doxorubicin resistance in hepatocellular carcinoma via modulating PTENPIJK/Akt pathway. J Cell Biochem. 2017;118(12):4498-507.

135. Xiong H, Ni Z, He J, Jiang S, Li X, He J, et al. LncRNA HULC triggers autophagy via stabilizing Sirt1 and attenuates the chemosensitivity of HCC cells. Oncogene. 2017;36(25):3528-40.

136. Yuan P, Cao W, Zang Q, Li G, Guo X, Fan J. The HIF-2alpha-MALAT1-miR-216b axis regulates multi-drug resistance of hepatocellular carcinoma cells via modulating autophagy. Biochem Biophys Res Commun. 2016;478(3):1067-73.

137. Jin W, Chen L, Cai X, Zhang Y, Zhang J, Ma D, et al. Long non-coding RNA TUC338 is functionally involved in sorafenib-sensitized hepatocarcinoma cells by targeting RASAL1. Oncol Rep. 2017;37(1):273-80.

138. Takahashi K, Yan IK, Wood J, Haga H, Patel T. Involvement of extracellular vesicle long noncoding RNA (linc-VLDLR) in tumor cell responses to chemotherapy. Mol Cancer Res. 2014;12(10):1377-87.

139. Yin $X$, Zheng $S S$, Zhang L, Xie $X Y$, Wang $Y$, Zhang BH, et al. Identification of long noncoding RNA expression profile in oxaliplatin-resistant hepatocellular carcinoma cells. Gene. 2017;596:53-88.

\section{Publisher's Note}

Springer Nature remains neutral with regard to jurisdictional claims in published maps and institutional affiliations.

\section{Ready to submit your research? Choose BMC and benefit from:}

- fast, convenient online submission

- thorough peer review by experienced researchers in your field

- rapid publication on acceptance

- support for research data, including large and complex data types

- gold Open Access which fosters wider collaboration and increased citations

- maximum visibility for your research: over $100 \mathrm{M}$ website views per year

At BMC, research is always in progress.

Learn more biomedcentral.com/submissions 